\title{
Consideraciones en el entrenamiento de habilidades psicológicas en esgrima
}

\section{Considerations for training in psychological skills in fencing}

\section{Consideraçôes nos treinamentos das habilidades psicológicas em esgrima}

\author{
Cristina Reche-García ${ }^{1}$, Mar Cepero-González ${ }^{2}$ y Francisco Javier Rojas-Ruíz ${ }^{2}$ \\ ${ }^{1}$ Universidad Católica San Antonio de Murcia $y^{2}$ Universidad de Granada
}

\begin{abstract}
Resumen: El objetivo de estudio fue conocer si existen diferencias en las habilidades psicológicas del esgrimista de competición en función de su nivel deportivo, edad y género. Fueron evaluados 90 esgrimistas (42 mujeres y 48 hombres; 20 élite y 70 no-élite; edad: $M=21.20$, dt= 7.01) sin entrenamiento psicológico previo. Se aplicó la Prueba para Evaluar Rasgos Psicológicos en Deportistas Versión Revisada (PAR P1-R; Serrato, 2006). Un MANOVA mostró diferencias significativas por nivel deportivo (Lambda de Wilks=0,79, $\mathrm{F}=2,58, \mathrm{p}=0,02$, Eta2 $=0,21)$ presentando mayor dominio en habilidades de confianza $(p=0,03)$, visualización $(p=0,03)$ y actitud positiva $(p=0,00)$ la élite frente a la no-élite. No se encuentran diferencias estadísticamente significativas en las variables evaluadas en función del género o edad. Estos resultados facilitan la selección y ajuste de estrategias psicológicas específicas en los planes de entrenamiento de esgrimistas con el objetivo de mantener y fortalecer sus habilidades psicológicas llegando a conseguir que se manifiesten al máximo nivel.

Palabras clave: Evaluación deportiva, habilidades psicológicas, esgrima, nivel deportivo, género, edad.

Abstract: The aim of study was to know if exist differences in the psychological skills in competition fencers around his athletic performance, age and gender. 90 fencers were evaluated ( 42 women and 48 men; 20 elite and 70 no-elite; age: $M=21,20, \mathrm{dt}=7,01)$ without previous psychological training. The evaluation system used was the Prueba para Evaluar Rasgos Psicológicos en Deportistas Versión Revisada (PAR P1-R; Serrato, 2006). A MANOVA showed significant differences in athletic performance (Lambda of Wilks=0,79, F= 2,58, p=0,02, Eta2=0,21), higher scores in skills
\end{abstract}

confidence $(p=0,03)$, imagery, $(p=0,03)$ and positive attitude $(p=0,00)$ in elite front no-elite. We didn't find significant difference in evaluated variables by gender or age. These results help to select and adjust specific psychological strategies in fencers training plans with the aim to keep and strengthen psychological skills getting their maximum level.

Keywords: Sportive evaluation, psychological skills, fencing, athletic performance, gender, age.

Resumo: O objetivo do estudo foi de conhecer se existem diferenças nas habilidades psicológicas do esgrimista de competição em função do seu nível esportivo, idade e gênero. Foram avaliados 90 esgrimistas (42 mulheres e 48 homens; 20 de elite e 70 de não - elite : idade: $M=21.20$, dt= 7.01) sem treinamento psicológico prévio. Foi aplicado a Prova para Avaliar Rasgos Psicológicos em Esportistas Versão Revisada (PAR P1-R; Serrato, 2006) Um MANOVA mostrou diferenças significativas por nível esportivo (Lambda de Wilks=0,79, F= 2,58, $\mathrm{p}=0,02$, Eta2 = 0,21), apresentando maior domínio nas habilidades de confiança $(p=0,03)$, e atitude positiva $(\mathrm{p}=0,00)$ entre a elite e não - elite. Não foi encontrada diferenças estatisticamente significativas nas variáveis avaliadas em funçáo do gênero e da idade. Estes resultados facilitam a seleção e o ajuste das estratégias psicológicas especifica nos planos de treinamentos do esgrimista com o objetivo de manter e fortalecer suas habilidades psicológicas podendo conseguir o máximo nível de manifestação.

Palavras Chave: Avaliação esportiva, habilidades psicológicas, esgrima, nível esportivo, gênero, idade.

\section{Introducción}

La identificación de los principales factores que inciden en el rendimiento de los deportistas de cada modalidad es el primer paso para diseñar una estrategia de entrenamientos específicos que mejore la efectividad del trabajo desarrollado por un deportista.

En todas las áreas de funcionamiento que implica el deporte de competición intervienen variables psicológicas que tienen una notable importancia, como son la motivación, la atención, el estrés, la ansiedad, la autoconfianza, los estados de ánimo, el autocontrol y la autorregulación, la cohesión, las habilidades interpersonales o el ajuste emocional, en la línea

Correspondencia: Facultad de Enfermería. Universidad Católica San Antonio. Campus de Los Jerónimos, s/n, 30107, Guadalupe, Murcia. España. Correo electrónico: creche@ucam.edu; Teléfono: +34968278808; Fax +34968278649 señalada por numerosos especialistas (Auweele, De-Cuyper, Van-Mele, y Rzewnicky, 1993; Buceta, 1990 y 1996; García, Rodríguez, Andrade y Arce, 2006; Gil, Capafons y Labrador, 1993; Highlen y Bennet, 1979; Loher, 1984; Mahoney, Gabriel, y Perkins, 1987; Meyers, Cooke, Cullen, y Liles, 1979; Ravizza, 1975; Roberts, 2001) (citado por Gimeno, Buceta y Pérez, 2007). El conocimiento de estas habilidades psicológicas en los deportistas condiciona la programación de sus entrenamientos.

En ocasiones tanto entrenador, como psicólogo deportivo, se plantean si adaptar el trabajo con sus deportistas en función de su nivel deportivo y edad (McCarthy, Jones, Harwood y Olivier, 2010) y adoptan diferentes roles, funciones y objetivos según estas características (Cox, 2009).

En la evaluación de habilidades psicológicas en deportistas se señalan diferencias en función del nivel deportivo, género y edad (Mahoney, Gabriel y Scott, 1987). 
En cuanto al género, se refieren diferencias en la variable confianza, siendo los hombres los que mayor puntúan. Así sucede en deportistas de distintas modalidades olímpicas (Lesser y Murphy, 1988), en nadadores (Balaguer, Escartí, Soler y Jiménez, 1990), en deportistas ecuestres (Meyers, Bourgeois, LeUnes y Murray, 1999), y en taekondistas (Carazo y Araya, 2010). Se añaden diferencias en la misma dirección para la variable concentración en deportes de pista y campo, voleibol, natación y baloncesto (Cox y Liu, 1993) y en practicantes de rodeo (Meyers, LeUnes, Bourgeois, 1996), siendo desestimadas en jugadores de bádminton (Bebetsos y Antoniou, 2003).

Aparecen estudios que señalan mayor motivación en deportistas mujeres que en hombres (Fortier, Vallerand, Briere y Provencher, 1995), con deportistas ecuestres (Meyers, Bourgeois, LeUnes y Murray, 1999), y en deportistas pertenecientes a distintos deportes (entre ellos esgrima), distinto nivel competitivo y edades (Balaguer, Castillo y Duda, 2007).

Por otro lado, atendiendo al nivel deportivo se presentan estudios que refieren diferencias en las habilidades psicológicas de los deportistas élite frente a no-élite en entrenamiento y competición en variables como la concentración, confianza y motivación en la evaluación de distintos deportes y edades (Mahoney, Gabriel, Perkins, 1987; Meyers, LeUnes, Bourgeois, 1996). En esta línea encontramos un estudio que halla diferencias en la variable concentración, destacando la élite en un grupo de deportistas de equitación (Meyers, Bourgeois, LeUnes y Murray, 1999). En discrepancia con un estudio de atención-concentración de esgrimistas, en el que no se halla una estrecha correlación con el rendimiento (Iglesias y Cano, 1990).

Distintas investigaciones concluyen una mayor confianza en élite frente a no-élite, en equitación femenina (Meyers, Bourgeois, Murray y LeUnes, 1993). Pero también, independientemente del género y en distintos grupos de deportes, entre ellos esgrima (Cox, Liu y Qiu, 1996). Además, mayor motivación en jugadores de hockey en la élite frente a la no-élite (Elferink-Gemser, Visscher, Lemmink y Mulder, 2004) y en levantadores de peso (Mahoney, 1989). Este último estudio en divergencia con otra investigación que señala mayor motivación en la no-élite en gimnasia rítmica (Jaenes, Carmona y Lopa, 2010).

Sin embargo, otros trabajos, afirman que no existen diferencias en las habilidades de los deportistas en función del género en variables tales como motivación, confianza, concentración, y visualización en futbolistas (Martín, 2003); tampoco en el dominio de distintas habilidades psicológicas en deportistas de raqueta y pala infanto-juveniles (Godoy, Vélez y Pradas, 2007); ni en función del nivel deportivo en judokas (Murphy, Fleck, Dudley y Callister, 1990) y mujeres tenistas (Meyers, Sterling, Bourgeois, Treadwell y LeUnes, 1994).

Ante este panorama de contradicciones y diferencias según deportes, práctica y contexto, surge la inquietud de explorar la situación actual de la esgrima en estas cuestiones.

La esgrima por sus características de deporte individual minoritario no ha recibido demasiada atención científica en comparación a otras disciplinas deportivas. Sin embargo, conocemos por investigaciones recientes que las habilidades psicológicas más destacadas de los esgrimistas son reto competitivo y sensibilidad emocional; precisando mejora en autoconfianza, motivación, actitud positiva y concentración; y especial atención en visualización. Además, que los esgrimistas con una mayor experiencia deportiva presentan una significativa mayor atención y control de sus emociones en detrimento de la motivación (Reche, Cepero y Rojas, 2010). En relación a la élite, los junior (menores de 20 años) muestran mayor dominio en motivación que los senior (mayores de 20 ańos), y estos últimos presentan deterioro de la concentración; no encontrándose diferencias por género (Reche, Cepero y Rojas, 2012).

Lo que no se conoce actualmente es si sería necesario diferenciar el trabajo con esgrimistas en función de su nivel deportivo, de su género y/o edad. Cuestión fundamental para facilitar la elaboración de programas de entrenamiento ajustados a las demandas psicológicas de cada esgrimista; y para aportar conocimiento a las maestrías de sus entrenadores, contribuyendo al desarrollo de la esgrima y a la preparación de sus practicantes. Es así que, el objetivo de este estudio es conocer si existen diferencias en las variables psicológicas evaluadas con el PARP1 R entre esgrimistas que conforman la élite y el resto de competidores, y en función de su edad y género.

\section{Método}

\section{Participantes}

La muestra está formada por 90 esgrimistas participantes asiduos del ranking nacional espańol en la modalidad deportiva de espada (modalidad más escogida y con mayor número de participantes). Es decir, esgrimistas que asisten regularmente a todas las competiciones en las que la edad no está limitada y cuya afluencia aproximada es de 47 mujeres y 81 hombres.

Se trata de 42 mujeres y 48 hombres con edades comprendidas entre los 15 y 40 ańos $(M=21.20, d t=7.01)$. A continuación se expone una tabla de contingencia con el número de deportistas según género en función de los tramos de edad y nivel deportivo (Tabla 1). 
Tabla 1. Número de deportistas en función de los tramos de edad, género y nivel deportivo.

\begin{tabular}{lllllllll}
\hline Género & \multicolumn{7}{l}{ Nivel deportivo } & \multicolumn{7}{l}{ Rangos de edad } \\
\hline \multirow{3}{*}{ Mujer } & No-élite & élite & Total & $15 / 17$ & $17 / 20$ & $\mathbf{2 0 / 3 0}$ & $30 / 40$ & Total \\
\cline { 2 - 9 } Hombre & 30 & 12 & 42 & 20 & 9 & 6 & 7 & 42 \\
Total & 40 & 8 & 48 & 24 & 6 & 9 & 9 & 48 \\
\hline
\end{tabular}

La élite está compuesta por seleccionados para representar a España en competiciones internacionales en la temporada 2009/2010, tanto senior como junior (con criterio de pertenecer a los 8 primeros del ranking junior y/o senior).

La no-élite está formada por participantes asiduos del ranking nacional español que al menos asistieron a 3 de los 4 campeonatos nacionales en la temporada previa en mujeres y a 5 de los 6 campeonatos en hombres.

La media semanal de sesiones de entrenamiento para la totalidad de la muestra es de 4,48 sesiones $( \pm 2,10)$ y la experiencia deportiva oscila entre menos de 1 año y más de 10 años, siendo 31 esgrimistas los que tienen hasta 5 años de experiencia deportiva y 59 los más expertos.

Ninguno de ellos ha participado en ningún programa de entrenamiento psicológico previo.

\section{Variables e instrumentos}

Las variables estudiadas para esta investigación fueron las habilidades psicológicas de los deportistas: Motivación, concentración, reto competitivo, actitud positiva, sensibilidad emocional, confianza y visualización de deportistas en contexto de entrenamiento y competición. Además, el género, edad y nivel deportivo (élite/no-élite). Para su estudio se recogió la información con la aplicación de la Prueba para Evaluar Rasgos Psicológicos en Deportistas (PAR P1-R; Serrato, 2006) elaborada específicamente para la evaluación psicológica en el ámbito deportivo y que incluye datos socio demográficos y deportivos. Su aplicación está dirigida a mayores de 12 ańos con un nivel cultural suficiente para comprender las instrucciones y poder ejecutar la tarea que se le exige. Presenta un total de 45 ítems que recogen información sobre el nivel de dominio de 7 variables implicadas en el rendimiento deportivo, confianza (con dos factores de segundo orden: actitud positiva y reto), motivación, concentración, visualización y sensibilidad emocional, obteniendo puntuaciones directas que se corresponden con puntuaciones estandarizadas (decatipos). Los decatipos se comparan con el perfil ideal y así es posible interpretar los resultados identificando debilidades en las variables psicológicas de los atletas a intervenir por el entrenador psicológico.

El cuestionario muestra unas propiedades apropiadas de fiabilidad y sensibilidad con un alfa de Cronbach de 0.90 en la medida completa y para los factores entre 0.705 y 0.929 , cumpliendo con los requisitos psicométricos necesarios para una evaluación deportiva fundamentada. Esta estandarizado y validado para evaluar habilidades psicológicas en deportistas (Serrato, 2006).

El diseño es cuasi experimental, descriptivo, de carácter transversal, siendo el género (masculino y femenino) el criterio seguido para confeccionar los grupos en un caso, y el nivel deportivo (élite y no-élite) y los tramos de edad en los otros.

\section{Procedimiento}

Para la recogida de datos se solicitó autorización a la Real Federación Española de Esgrima para que con motivo de los primeros campeonatos de la temporada 2009/2010 se nos permitiese el reparto de los cuestionarios y la solicitud de participación voluntaria entre los espadistas del ranking nacional absoluto. De esta forma, se reunieron a todos los maestros nacionales en el desarrollo de una prueba de ranking, se les explicó y describió la investigación y la forma de cumplimentación de la prueba psicológica pidiéndoles su colaboración para el reparto entre sus alumnos. Se señaló la importancia de que la aplicación fuese llevada a cabo exclusivamente por espadistas asiduos al ranking nacional absoluto previo (con asistencia total al circuito o una sola ausencia) y de forma voluntaria. Se instó a que consultasen cualquier tipo de duda que les pudiese surgir y que devolviesen al directorio técnico (organismo encargado de la dirección del torneo compuesto por responsables informáticos y una delegación arbitral) los cuestionarios antes de retirarse de las instalaciones deportivas.

Todos los participantes cumplimentaron la prueba de forma individual a lo largo de su campeonato (en sus tiempos muertos) y firmaron su consentimiento informado. El procedimiento fue aprobado por el Comité de Ética de la Universidad de Granada.

\section{Análisis de los datos}

El análisis de los datos se efectúa a partir de estadísticos descriptivos y de contraste de comparación de medias (análisis de varianza multivariado MANOVA), mediante el paquete estadístico SPSS versión 18.0 para Windows. A efectos de interpretación y análisis de los datos el nivel de confianza asumido fue de $0.05(\mathrm{p}<0.05)$. 


\section{Resultados}

Con el fin de conocer la diferencia de medias debidas al género, rango de edades y nivel deportivo en el nivel de dominio de las habilidades psicológicas evaluadas, se realiza una MANOVA en el que se introducen como variables dependientes las puntuaciones del PARP1 R y como variables independientes el género, nivel deportivo y rango de edad. El análisis de contrastes multivariados señala diferencias estadísticamente significativas entre los vectores de medias en función del nivel deportivo (Lambda de Wilks=0,79, $\mathrm{F}=2,58, \mathrm{p}=0,02$, Eta2 $=0,21$, no siendo así en función del género (Lambda de Wilks $=0,91, F=0,93, p=0,48, E t a 2=0,08)$, ni por rangos de edad (Lambda de Wilks=0,64, F=1,53, p=0,07, Eta2= $0,13)$. El tamańo del efecto para el nivel deportivo es mediano (eta $>0,2)$.

Tabla 2. Prueba de los efectos intersujetos para el grupo élite/no-élite

\begin{tabular}{lllll}
\hline & $\mathrm{MC}$ & $\mathrm{F}$ & $\mathrm{p}$ & $\mathrm{Eta2}$ \\
\hline Confianza & 9,59 & 4,78 & $0,03^{*}$ & 0,06 \\
Motivación & 4,29 & 1,40 & 0,24 & 0,01 \\
Concentración & 12,29 & 2,55 & 0,11 & 0,03 \\
Sensibilidad emocional & 0,45 & 0,09 & 0,76 & 0,00 \\
Imaginación & 17,29 & 4,82 & $0,03^{*}$ & 0,06 \\
Actitud positiva & 26,55 & 8,33 & $0,00^{*}$ & 0,10 \\
Reto & 0,16 & 0,00 & 0,93 & 0,00 \\
${ }^{*} p<0.05$ & & & &
\end{tabular}

Comparando medias en función del nivel deportivo del esgrimista, encontramos diferencias estadísticamente significativas $\left({ }^{*} p<0.05\right)$ en las variables confianza $(p=0.03)$, imaginación $(p=0.03)$ y actitud positiva $(p=0.00)$ entre el grupo que pertenece a la élite y el no-élite. El tamaño del efecto para confianza (eta2 $=0.06)$ e imaginación (eta2 $=0.06$ ) es mediano y para actitud positiva (eta $2=0.10)$ es pequeño.

\section{Discusión y conclusiones}

La evaluación realizada no muestra diferencias estadísticamente significativas en función del género en las respuestas del PAR P1-R ofrecidas por los esgrimistas asiduos al ranking nacional espańol, resultados afines a los de Martín (2003) y Godoy y cols. (2007) y contrarios a los trabajos que si las encuentran en distintos deportes y grupos de edad, en variables como motivación (Fortier y cols., 1990; Meyers y cols., 1999; Balaguer y cols., 2007); confianza (Lesser y Murphy, 1988; Balaguer y cols. 1990; Cox y Liu, 1993; Meyers y cols. 1996; Meyers y cols., 1999; y Carazo y Araya, 2010); o concentración (Cox y Liu, 1993; y Meyers y cols., 1996). Diferencias que bien pueden deberse a las exigencias específicas e intrínsecas de un deporte, contexto socio-cultural determinado y el rol asignado a la mujer en ese momento.

En relación a la edad no se encuentran diferencias por rangos de edad en las habilidades psicológicas de los participantes en el estudio, en consonancia con Reche y cols. (2012) para esgrimistas élite. Es de señalar que la edad no es lo mismo que experiencia deportiva, para la que si se han encontrado diferencias en las competencias psicológicas de los esgrimistas (Reche y cols., 2010).

Indudablemente, el nivel competitivo está asociado a un entrenamiento de mayor calidad con desarrollo de destrezas que repercute en el rendimiento. En nuestro trabajo se encuentra que la élite posee mayor confianza, actitud positiva y capacidad de visualización que la no-élite en entrenamiento y competición. En divergencia con estudios que no encuentran diferencias en función del nivel deportivo en distintos deportes y con participantes de distintas edades (Murphy y cols., 1990; y Meyers y cols. 1994).

La élite de esgrima presenta mayor confianza frente a la no-élite, ratificando distintos estudios (Meyers y cols., 1993; Cox y cols., 1996; Meyers y cols. 1996; Mahoney y cols. 1997). Mayor actitud positiva (factor de segundo orden de la variable confianza), habilidad que tiene el deportista para mantener la confianza antes y durante los momentos difíciles de la competición, así como para reflejar ante los demás una actitud de un deportista ganador. Además, mayor capacidad de visualización, confirmando el estudio de Gregg y Hall (2006) en distintos tipos de deporte.

En cuanto a la variable motivación, no encontramos diferencias en los deportistas evaluados, en contra de estudios que si lo hacen (Meyers y cols.1996; Mahoney y cols. 1989; Elfeink-Gemser y cols., 2004; Jaenes y cols., 2010). Tampoco para la variable concentración-atención, ratificando el trabajo de Iglesias y Cano (1990), en el que no se halla una estrecha correlación entre atención-concentración y el rendimiento en un grupo de esgrimistas españoles.

La revisión de la literatura nos muestra perfiles psicológicos específicos en función del deporte y su profesionalidad, participantes (género, edad) y contexto socio-cultural. Así como por las demandas del deporte, recompensas y requerimientos que lo caracteriza. En el caso de la esgrima española, concluimos la existencia de diferencias en habilidades y competencias psicológicas en función del nivel deportivo, pero no en función del género y edad. El grupo de alto rendimiento o élite de esgrimistas presenta mayor dominio en habilidades de confianza, visualización, y actitud positiva, en comparación con el grupo no-élite. Por ello, podríamos considerar a estas variables psicológicas como determinantes psicológicos del rendimiento deportivo en esgrima, recomendar su atención para aumento de su rendimiento, así como no diferenciar el tratamiento y trabajo por edad y género. 
El psicólogo deportivo de esgrimistas y los maestros de armas en España debieran tener en cuenta las conclusiones del estudio en el diseño de los planes de entrenamiento para llegar a conseguir el perfil de rendimiento óptimo en sus deportistas y un mejor afrontamiento a la competición. Se trata de optimizar las competencias del deportista, favoreciendo sus sistemas de preparación, beneficiando, sin duda, el desarrollo y perfeccionamiento del resto de habilidades deportivas: físicas, técnicas y táctico-estratégicas.

Sobre la base de esta información se propone ofrecer a las escuelas deportivas y tecnificación deportiva una intervención psicológica a esgrimistas con una planificación estructurada de un programa de entrenamiento psicológico para la próxima temporada. Además, unas jornadas formativas dirigidas a informar a los Maestros de Armas acerca del desarrollo y aprendizaje de destrezas psicológicas de sus atletas. Y como futura línea de investigación un estudio transcultural que revise diferentes contextos socioculturales en los que se desarrolla la práctica deportiva de esgrima.

\section{Bibliografia}

1. Auweele, Y. V., De-Cuyper, B., Van-Mele, V. y Rzewnicky, R. (1993). Elite Performance and Personality: From Description and Prediction to Diagnosis and Intervention. En R. N. Singer, M. Murphey y L. K. Tennant (eds.): Handbook of Research on Sport Psychology (pp. 257-299). New York: Macmillan.

2. Balaguer, I., Castillo, I. y Duda J. L. (2007). Psychometric properties of the sports motivation scale in Spanish Athletes. Revista Mexicana de Psicología, 24(2), 197-207.

3. Balaguer, I., Escartí, A., Soler, M. J. y Jiménez, C. (1990). Influencia de la autoconfianza en el deporte y de la orientación competitiva sobre la ejecución en un grupo de nadadores orientados a la competición: $I I$ Congreso del colegio oficial de Psicólogos, 90-95, Valencia, Abril, Colegio oficial de psicólogos de Madrid.

4. Bebetsos, E. y Antoniou, P. (2003). Psychological skills of Greek Badminton athletes. Perceptual Motor Skills, 3(2),1289-96.

5. Buceta, J. M. (1990). Aspectos a tener en cuenta en relación con las deportistas españolas de alta competición. Seminario Mujer y Deporte, organizado por el Consejo Superior de Deportes, la Secretaría de Estado de Educación y el Instituto de la Mujer en Madrid. Publicado posteriormente en Planificación del Entrenamiento Deportivo y Rendimiento: Un enfoque interdisciplinar. Málaga: Instituto Andaluz del Deporte, 1995.

6. Buceta, J. M. (1996). Psicología y lesiones deportivas: Prevención y recuperación. Madrid: Dykinson.

7. Buceta, J. M. y Pérez, M. C. (2007). Influencia de las variables psicológicas en el deporte de competición: evaluación mediante el cuestionario Características psicológicas relacionadas con el rendimiento deportivo. Psicothema, 19(4), 667-672.

8. Carazo, P. y Araya, G. A. (2010). Perfil de rasgos psicológicos para el rendimiento deportivo en hombres y mujeres practicantes de taekwondo. Revista Iberoamericana de Psicología del Ejercicio y el Deporte, 5(2), 253-266.

9. Cox, R. H. y Liu, Z. (1993): Psychological skills: A cross-cultural investigation. International Journal of Sport Psychology, 24, 326-340.

10. Cox, R. H., Liu, Z. y Qiu, Y. (1996). Psychological skills of elite Chinese athletes. International Journal of Sport Psychology, 27(2), 123-132.

11. Cox, R. H. (2009). Psicología del deporte: Conceptos y sus aplicaciones (6 ${ }^{\mathrm{a}}$ ed). Madrid: Editorial Médica Panamericana.

12. Elferink-Gemser, M., Visscher, C., Lemmink, K. y Mulder, T. (2004). Relation between multidimensional performance characteristics and level of performance in talented youth field hockey players. Journal of Sports Sciences, 22(11-12), 1053-1063.

13. Fortier, M. S., Vallerand, R. J., Briere, N. M. y Provencher, P. J. (1995). Competitive and recreacional sport structures and gender: A test of their relationship with sport motivation. International Journal of Sport Psychology, 26(1), 24-39.

14. García, E. M., Rodríguez, M., Andrade, E. M. y Arce, C. (2006). Adap- tación del cuestionario MSCI para la medida de la cohesión en futbolistas jóvenes españoles. Psicothema, 18(3), 668-672.

15. Gil, M., Capafons, B. y Labrador, E. (1993). Variables físicas y psicológicas predictoras del rendimiento deportivo y del cambio terapéutico. Psicothema, 5(1), 97-110.

16. Gimeno, F., Buceta, J. M. y Pérez, M. C. (2007). Influencia de las variables psicológicas en el deporte de competición: evaluación mediante el cuestionario Características psicológicas relacionadas con el rendimiento deportivo. Psicothema, 19(4), 667-672.

17. Godoy, D., Vélez, M. y Pradas, F. (2007). Nivel de dominio de las habilidades psicológicas en jóvenes jugadores de deportes de raqueta y pala: tenis de mesa y bádminton. Cuadernos de Psicología del Deporte, 7(1), 45-59.

18. Gregg, M. y Hall, C. (2006). Measurement of motivational imagery abilities in sport. Journal of Sports Sciences, 24(9), 961-971.

19. Highlen, P. S. y Bennett, B. B. (1979). Psychological characteristics of successful and nonsuccessful elite wrestlers: An exploratory study. Journal of Sport Psychology, 1, 123-137.

20. Iglesias, X. y Cano, D. (1990). El perfil del esgrimista en Catalunya. Apunts: Educació Física i Esports, 19, 45-54.

21. Jaenes, J. C., Márquez, J. C. y Lopa, E. (2010). Revista Iberoamericana de Psicología del Ejercicio y del Deporte, 5(1), 15-28.

22. Lesser, M. y Murphy, S. M (1988). The psychological skills inventory for sport (PSIS): Normative and reliability data. Paper presented at the anual meeting of the American Psychological Association, Atlanta, GA.

23. Loehr, J. E. (1984). How to overcome tension and play at your peak all the time. Tennis, 19, 66-76. Madrid. Dykinson.

24. Mahoney, M. J., Gabriel, T. J. y Perkins, T. S. (1987). Psychological skills and exceptional athletic performance. The Sport Psychologist, 1, 181-199.

25. Mahoney, M. J (1989). Psychological predictors of elite and non-elite performance in Olympic weightlifting. International Journal of Sport Psychology, 20, 1-12.

26. Martín, G. (2003). La mujer futbolista desde la perspectiva psicológica. Cuadernos de Psicología del Deporte, 3, 7-15.

27. McCarthy, P. J., Jones, M. V., Harwood, C. G. y Olivier, S. (2010). What do young athletes implicitly understand about psychological skills?. Journal of Clinical Sport Psychology, 4, 158-172.

28. Meyers, A. W., Cooke, C. J., Cullen, J. y Liles, L. (1979). Psychological aspects of athletic competitors: A replication across sports. Cognitive Therapy and Research, 3, 361-366.

29. Meyers, M. C., Bourgeois, A. E., Murray, N. y LeUnes, A. (1993). Comparison of psychological characteristics and skilld of elite and sub-elite equestrian athletes. Medicine and Science in Sport and Exercise, 25, S154.

30. Meyers, M. C., Sterling, J. C., Bourgeois, A. E., Treadwell, S. y LeUnes, A. (1994). Mood and psychological skills of elite, world-ranked female tennis players. Journal of Sport Behavior, 17, 156-165. 
31. Meyers, M. C., LeUnes, A. y Bourgeois, A. E (1996). Psychological skills assessment and athletic performance in collegiate rodeo athletes. Journal of Sport Behavior. 19 (2), p. 132-146

32. Meyers, M. C., Bourgeois, A. E., LeUnes, A. y Murray, N. G. (1999). Mood and psychological skills of elite and sub-elite equestrian athletes. Journal of Sport Behaviour, 22 (3), 399-409.

33. Murphy, S. M., Fleck, S. J., Dudley, G. y Callister, R. (1990). Psychological and performance concomitants of increased volumen training in elite athletes. Journal of Applied Sport Psychology, 2, 34-50.

34. Ravizza, K. (1975). A subjective study of the athlete's greatest moment in sport. Proceedings of the 7th Canadian Psychomotor Learning and Sport Psychology Symposium
35. Reche, C., Cepero, M. y Rojas, F. J. (2010). Efecto de la experiencia deportiva en las habilidades psicológicas de esgrimistas del ranking nacional. Español. Cuadernos de Psicología del Deporte, 10(2), 33-42.

36. Reche, C., Cepero, M. y Rojas, F. J. (2012). Perfil psicológico en esgrimistas de alto rendimiento. Cultura, Ciencia y Deporte, 19(7), 35-44.

37. Roberts, G. C. (Ed.) (2001). Advances in motivation in sport and exercise. Champaign, IL: Human Kinetics.

38. Serrato, L. (2006). Revisión y estandarización de la prueba elaborada para evaluar rasgos psicológicos en deportistas (PAR-P1) en un grupo de deportistas de rendimiento en Colombia. Cuadernos de Psicología del Deporte, 6(2), 67-84. 